\title{
KAZN As a Diagnostic Marker in Ovarian Cancer: A Comprehensive Analysis Based on Microarray, MRNA-Sequencing, and Methylation Data
}

\section{Songling Zhu}

Harbin Medical University

Hongxia Bao

Harbin Medical University

Mengchun Zhang

Harbin Medical University

Xingjuan Zhao

Harbin Medical University

Shu-lin Liu ( $\nabla$ slliu@hrbmu.edu.cn )

Harbin Medical University

\section{Research Article}

Keywords: KAZN, ovarian cancer, methylation, bioinformatics, survival

Posted Date: May 28th, 2021

DOI: https://doi.org/10.21203/rs.3.rs-515864/v1

License: (c) (1) This work is licensed under a Creative Commons Attribution 4.0 International License. Read Full License 


\section{Abstract}

Background: Previous studies have shown that KAZN is involved in multiple biological processes such as cell development, proliferation, differentiation, and apoptosis. Most of the studies related to KAZN have been carried out in keratinocytes. Apart from that, KAZN is also expressed in other tissues, such as the ovary. However, the related research is relatively few and the function in other tissue or cell is still not clear.

Methods: We investigated the correlations between KAZN expression and clinical characteristics of ovarian cancer (OC) and compared methylation levels of normal and OC samples through data collected from Gene Expression Omnibus (GEO) microarrays, The Cancer Genome Atlas (TCGA), and Clinical Proteomic Tumor Analysis Consortium (CPTAC). The relationships among differentially methylated sites in the KAZN gene, corresponding KAZN mRNA expression levels and prognosis were further analyzed.

Results: KAZN was up-regulated in ovarian epithelial tumors and the expression of KAZN was correlated with the patients' survival time. KAZN CpG site cg17657618 was positively correlated to the expression of mRNA and the methylation levels are significantly differential between the group of the stage of "I and II" the group of the stage "III and IV". This study also presents a method to classify tumor and normal tissue in OC using DNA methylation pattern in the KAZN gene body region.

Conclusions: We validated that KAZN was involved in ovarian cancer progression. These results may provide a new direction for ovarian cancer research and provide a potential diagnostic biomarker and therapeutic target.

\section{Background}

Ovarian cancer is one of the most lethal gynecological malignancies in women. Due to the absence of symptoms in the early stages and late detection, ovarian cancer is usually fatal. Data from SEER 18 2010-2016 show that 5-years relative survival for all patients with ovarian cancer is $46.8 \%$, but 5-years relative survival in patients with stage localized is significantly better than in patients with stage distant (respectively $92.6 \%$ and $30.2 \%$ )[1]. The most common histological types are epithelial tumors, including high-grade serous carcinoma (HGSOC, accounts for 60\%), endometrioid, clear cell, mucinous, and lowgrade serous carcinomas[2]. Recent studies report that the different histological subtypes of ovarian cancer may have distinct origins. Although a variety of molecular alterations in ovarian cancer have been reported, including genetic factors, such as $B R C A 1$ and $B R C A 2[3,4]$, and genes that are involved in DNA mismatch repair systems, such as $M S H 2, M S H 6$, and TP53, and other mutated genes and pathways[5-9]. Because of the diversity of molecular alteration and historical heterogeneity of ovarian cancer, the application of these studies for clinical diagnosis and effective therapy is rare and further investigation is required[10].

Epigenetic changes are integral to all aspects of cancer genomics. DNA methylation is one of the common epigenetic mechanisms, which can affect gene expression and other mechanisms that 
participate in the formation and development of cancer[11, 12]. A large body of study has shown that aberrant DNA methylation of global DNA or specific genes may affect the progression[13] and prognosis of ovarian cancer[14]. However, the mechanisms of how these aberrant DNA methylations are involved in ovarian cancer are still not yet clear.

$K A Z N$ is an evolutionarily conserved gene initially identified in keratinocytes and then considered to be widely expressed across different human tissues[15]. The cellular localization of $K A Z N$ changes dynamically during the development of mouse eggs[16]. KAZN is partially co-localized with desmoplakin and periplakin at desmosome and involved in the interplay between adherens junctions and desmosomes[15]. By binding to actin and intermediate filament, KAZN can affect the cell shape and remodeling of cytoskeletal networks[17]. In the nucleus, $K A Z N$ was found to be associated with the cell cycle, gene regulation, and matrix stability. KAZNinteracts with apoptotic regulators, ARC and Bax, and plays an important role in apoptosis and cell growth[18]. KAZN is up-regulated during keratinocyte terminal differentiation[17] and dispensable for murine epidermal morphogenesis and homeostasis[19]. Since $K A Z N$ is involved virtually in all aspects of cell development, it must play critical roles in the genesis and development of tumors[20,21]. According to the dynamic change of expression of the KAZN during cell development and the regulation of gene expression by epigenetics in time and space[22, 23], we postulate that the expression and methylation of $K A Z N$ are crucial in the occurrence and development of cancer. To validate this postulation, we conducted a comprehensive analysis based on GEO and TCGA data in ovarian cancer.

In this study, using the data from GEO, TCGA, GTEx, and CPTAC datasets, we compared KAZN expression in the varied level of mRNA, protein, and DNA methylation, and detected the correlation between expression and survival time. Our data indicated that the expression and methylation pattern of $K A Z N$ may play important role in the oncogenesis of ovarian cancer. These results can contribute to understanding the molecular mechanisms that are involved in tumor progression and can be used to develop new treatment strategies.

\section{Materials And Methods}

\section{Gene expression omnibus (GEO) database}

The GEO database (https://www.ncbi.nlm.nih.gov/geo/) is a public data storage repository of microarray, methylation, and next-generation sequencing data. We conducted searching in GEO datasets with keywords "ovarian cancer" and "mRNA" and then filtered by the following criteria: 1 organism from homo species, 2 contains the tumor and non-tumor tissues (normal, benign, or para-carcinomas tissue). $\mathrm{R}$ package 'GEOquery' was used for data download and preprocessing. R package 'Limma' was used to normalize and analyze the data. A t-test is a statistical test that is used to compare the means of two groups. R package 'ggpubr' was used to plot boxplot.

\section{Comprehensive meta-analysis}


R package 'meta' was used for performing a comprehensive meta-analysis of GEO data. The analysis of $K A Z N$ expression in the normal and tumor group was displayed on forest plots that illustrate the standardized mean difference (SMD) and the $95 \%$ confidential interval $(\mathrm{Cl})$. The chi-squared test of Q and the 12 statistic was calculated to assess heterogeneity across the studies and to determine the appropriateness of applying either a random-effects model or fixed-effects model to the pooling process. To measure publication bias, Egger's and Begg's tests and a funnel plot, for which significance was $p<$ 0.05 , were performed.

\section{TCGA, GTEx, and GEO gene expression data integration and differentially expressed genes (DEGs) identification}

RNA-Seq data of 356 ovarian cancer and 180 normal tissue samples were downloaded from GenotypeTissue Expression (GTEx) (https://www.gtexportal.org/home/) and TCGA via NCl's Genomic Data Commons (GDC) portal (https://portal.gdc.cancer.gov/). Besides, five gene expression profiles - Datasets GSE137238, GSE101108, GSE101948, GSE143897, and GSE132107 - were downloaded from GEO, with a sample size of 323 ovarian cancer and 8 normal tissue samples. We integrated the data and ran 'ComBat-seq' in the R package 'SVAseq' (Version 3.38;

http://bioconductor.org/packages/release/bioc/html/sva.html) to correct the batch effect. 'DESeq2' (Version 1.30.1[24]; http://bioconductor.org/packages/release/bioc/html/DESeq2.html) was used to detect the differential gene expression between ovarian cancer and normal ovarian tissue samples. The expression of $K A Z N$ in each sample was plotted with the $\mathrm{R}$ 'circlize' package (Version 0.4.12; https://cran.r-project.org/web/packages/circlize/index.html).

\section{Proteomic analysis:}

Proteomic data were downloaded from The National Cancer Institute's Clinical Proteomic Tumor Analysis Consortium (CPTAC) (https://proteomics.cancer.gov/programs/cptac)[25]. After quality evaluation, a total of 84 ovarian cancer tissue and 22 normal tissue samples from the CPTAC Ovarian Cancer Confirmatory Study was retained for further proteomic study. Differentially expressed proteins (DEPS) between normal and tumor tissues were identified by R package 'Limma' with a FDR $<0.05$ cut-off criteria.

\section{Survival Analysis}

Overall survival was computed as the number of years between the year of diagnosis and the year of death from all causes, the date of the last follow-up, or 5-year censored survival data. Kaplan-Meier curves comparing overall survival according to subgroups divided by expression of $K A Z N$ and a log-rank test was used to assess the survival distributions across the subgroups. Samples were divided into either "high" or "low" groups with the cutoff at the lower quartile and upper quartile of KAZN gene expression. Gene Expression Profiling Interactive Analysis (GEPIA) (http://gepia.cancer-pku.cn) provides interactive analyses of cancer and normal genes. To further clarify the relationship between $K A Z N$ expression and OC prognosis, we also used GEPIA for survival analysis and log-rank for statistical analysis. In log-rank, 
$p<0.05$ was considered statistically significant. The survival curve was plotted by R package 'survival' and 'survminer'.

\section{Methylation level and mRNA expression level correlation analysis}

The DNA methylation data (Illumina Human Methylation 27k) of ovarian cancer $(n=601)$ and normal tissues $(n=12)$ and corresponding clinical information were obtained from TCGA (https://portal.gdc.cancer.gov/). The obtained DNA methylation data were further analyzed using Perl script and R package 'ggpubr'. The Pearson correlation coefficient test was used to examine statistical significance in differences between DNA methylation and the expression of KAZN. R package 'ggplot2' was used to plot.

\section{$K A Z N$ gene methylation pattern analysis}

GEO datasets Illumina Infinium 450K BeadChips were used to detect the methylation sites. R package 'minfi' was used to check the different CpGs sites. Online wANNOVAR webserver was used to annotate the CpGs sites[26]. R package 'pheatmap' was used to generate the figure.

\section{Results}

\section{Assessment of $K A Z N$ mRNA level in ovarian cancer, based on gene expression omnibus (GEO) datasets}

The expression data of $K A Z N$ in ovarian cancer were obtained through the GEO database. A total of 10 microarrays from the GEO database met the entry criteria. The features of the selected GEO datasets are depicted in Table 1. Of all the data sets, 9 were source tissue samples, one of which was a blood sample. Expression of KAZN was significantly increased in ovarian cancer tissues in GSE105437, GSE18520, GSE27651, GSE36668, GSE38666, GSE40595, GSE66957, GSE69428 $(p=0.0017, p=0.0012, p<0.0001$, $p=0.0075, p<0.0001, p<0.0001, p<0.0001, p=0.0177$, respectively) (Figure 1). But no statistical difference was detected in GSE29450 ( $p=0.0549)$ and down-regulation of the expression of KAZN was found in normal group in GSE37582 ( $<<0.001$, supplemental file: Figure $S 1)$.

\section{Meta-analysis of GEO datasets}

A meta-analysis was conducted based on 10 included microarrays from the GEO database. The results are demonstrated in Figure 2A. Given the apparent heterogeneity $(p<0.01,12=91 \%)$, a random-effects model was applied, and remarkable up-regulation $(S M D=0.99,95 \% \mathrm{Cl}: 0.12,1.86)$ of $K A Z N$ mRNA was found in ovarian cancer group.

Sensitivity analysis was performed to explore whether a particular microarray played a vital role in significant heterogeneity (Figure 2B). By removing an individual study per time of meta-analysis to assess the influence of each study, the result showed that no study was found to have played a crucial role in any of the enrolled studies. A funnel plot showed that no evidence of publication bias was observed for this analysis ( $p=0.6547$, Figure $2 C)$. 


\section{Up-regulation of $K A Z N$ affects overall survival in ovarian cancer}

Since TCGA ovarian cancer datasets have only cancer tissue samples, no normal tissue as a control, so we compare them to GTEx samples, which have expression data from normal ovary tissue of GTEx donors who don't have cancer. To eliminate the batch effect, we conducted ComBat-seq to integrate different sourced datasets[24]. The comprehensive analysis based on TCGA, GTEx, and GEO datasets showed that the KAZN mRNA expression is significantly differential $(p<0.001)$ (Figure 3$)$, and the expression was up-regulated in the TCGA group compared to GTEx group $(p<0.0001$, Figure 4A).

To further study the clinical effects of $K A Z N$ in ovarian cancer, all cases were divided into two groups ( $K A Z N$ high expressed and $K A Z N$ low expressed) by the quantiles of $K A Z N$ counts. Then we analyzed the survival status between the two groups. A Kaplan-Meier curve was used to identify the effects of the expression of $K A Z N$ on survival time. The result showed that in $K A Z N$ low expressed group survival time is significantly longer than in the KAZN high expressed group (Figure 4B). The results based on the Gepia2 (http://gepia2.cancer-pku.cn/\#survival) online website analysis confirm our above analysis (supplemental file: Figure S2).

\section{$K A Z N$ protein is Up-regulated in ovarian cancer}

The Clinical Proteomic Tumor Analysis Consortium (CPTAC) has produced huge amounts of cancer proteomics data providing unprecedented research opportunities. The dataset from CPTAC Ovarian Cancer Confirmatory Study contains 41 normal participants and 169 tumor participants, was used to validate the KAZN protein expression in Ovarian cancer. The result showed that in the tumor group, the KAZN protein expression was significantly higher than in the normal group ( $p=0.0027$, Figure 5). KAZN protein expression was consistent with mRNA level in this study.

\section{$K A Z N$ methylation level is correlated to mRNA expression in ovarian cancer}

Methylated CpG sites have a moderate/strong association with gene expression changes across the phases in cancer-involved genes with specific functions. To study the correlation between the expression of $K A Z N$ and DNA methylation, we detected the DNA methylation level of $\mathrm{CpG}$ sites in the $K A Z N$ gene body region in TCGA datasets. From the TCGA database, we obtained 27K DNA methylation array data of ovarian cancer which contains 601 tumors and 12 normal samples. 27K methylation array covers only 2 CpG sites for KAZN, respectively is cg21045388 and cg17657618. The cg21045388 is highly methylated in almost all samples. So we detected the correlation between the expression of KAZN and cg17657618. We found the methylated $\mathrm{CpG}$ site cg17657618 positively correlated with the rising expression of the KAZN gene (Figure 6A).

To detect the correlation between $K A Z N D N A$ methylation and progress of $\mathrm{OC}$, we divided all cases into two groups by stage of clinical traits. We compared the methylation level between the two groups. The result showed that the methylation level of the stage "I and II" group is significantly lower than the stage 
"III and IV" group (Figure 6B). The results suggested that KAZN methylation may have an important role in the development and outcome of ovarian cancer.

\section{The diagnostic value of $K A Z N$ methylation status in ovarian cancer}

Illumina Infinium 450K BeadChip covers 134 CpG sites for KAZN. Based on the GSE146552 dataset, we found 13 differentially methylated $\mathrm{CpG}$ sites in the $K A Z N$ gene body region (details in supplemental table S1). Among these differentially methylated CpG sites, six were hypermethylated and seven were hypomethylated. Interestingly, an unsupervised hierarchical clustering based on the 13 different methylated CpG sites in KAZN almost perfectly divided the samples of GSE146552 into two groups, the EOC (Epithelial ovarian cancer) cluster, and the NE (Not Epithelial OC) cluster (Figure 7). The above results suggest that this CpGs pattern may as a new biomarker contribute to the diagnosis of ovarian cancer.

\section{Discussions}

Previous studies have shown that $K A Z N$ is involved in multiple biological processes such as cell development, proliferation, differentiation[17], and apoptosis[18], which naturally raise us to associate $K A Z N$ with tumor formation and metastasis. Most of the studies related to $K A Z N$ have been carried out in keratinocytes $[15,17,27]$. Apart from that, $K A Z N$ is also expressed in other tissues, such as the ovary. However, the related research is relatively few and the function in other tissue or cell is still not clear. $A$ study has been reported that $K A Z N$ is associated with cervical cancer[28], but the correlation between $K A Z N$ and $\mathrm{OC}$ has not been investigated.

In the present study, we found that the expression of $K A Z N$ is significantly associated with $O C$. We compared 10 GEO microarray datasets to detect $K A Z N$ expression at mRNA level and validate the results using a meta-analysis. KAZN mRNA is significantly up-regulated in OC in 8 GEO datasets. But such results were not seen in two GEO datasets, one from clear cell cancer and one from blood. According to the GTEx Transcript Browser analysis, KAZN transcripts exhibit the same expression pattern in epithelial cells[29]. So, the expression inconsistencies in $K A Z N$ may be due to different tissue sources.

Using multiple data sources, we confirmed that $K A Z N$ was differentially expressed both on the mRNA level and on the protein level between ovarian cancer and normal tissues. And the KAZN mRNA expression was negatively correlated with survival time. The results confirmed that $K A Z N$ was critical to the oncogenesis and development of ovarian cancer. KAZN can dually regulate proliferation and differentiation by Rho-dependent and -independent mechanisms[17]. KAZN plays an important role in regulating cellular apoptosis by interacting with ARC and Bax[18]. Also, during invasion and metastasis, cancer cells undergo changes in morphology and disruptions of cell connections. $K A Z N$ can regulate cytoskeletal networks and cause changes in cell shape, and as a component of the desmosome, KAZN participates in the formation of cell connections[15, 17]. In light of this, we speculate $K A Z N$ gene may participate in tumorigenesis, invasion, and metastasis of $\mathrm{OC}$ by affecting these key processes. 
Growing evidence has shown that epigenetic changes play a critical role in cancer development and progression[22]. DNA hypermethylation can suppress the expression of tumor suppressor genes and hypomethylation can induce genomic instability[30]. There was a study that revealed the association of CpG site cg17657618 with Endometriosis[31], but no reports about this $\mathrm{CpG}$ site in ovarian cancer. Our results based on TCGA human methylation 27K BeadChips validated that cg17657618 was hypermethylated and positively correlated with the expression of the KAZN in OC. Based on the $450 \mathrm{~K}$ array dataset GSE146552, we found another differentially expressed 13 methylation sites. The differentially expressed CpG sites, included 6 hypermethylated, and 7 hypomethylated in OC samples, may affect the expression of transcripts of $K A Z N$ in different ways, and further participated in the formation and progression of ovarian cancer. These results further confirmed the $K A Z N$ gene expression and its methylation were critical in $\mathrm{OC}$ occurrence and progress.

It is common to use cluster analysis to divide patients into different groups based on the expression value of multiple genes or the top-ranked methylation $\mathrm{CpG}$ sites $ß$-value. However, our results based on single gene methylation $\mathrm{CpG}$ site $ß$-value were surprising. The methylation $\mathrm{CpG}$ site pattern of $K A Z N$ could be a new biomarker for predicting and diagnosing $\mathrm{OC}$ and a potential therapeutic target. KAZN contains multiple transcripts, some of them are tissue-specific and expressed in a temporal sequence, these transcripts may play different roles at different stages in the development of ovarian cancer. Further understanding of the function of the $K A Z N$ gene and the mechanistic relationship between $K A Z N$ expression and methylation in cancer is needed.

\section{Declarations}

\section{Ethics approval and consent to participate}

The data of breast cancer patients we used in this study are all from the publicly accessible database. Separate ethics committee approval is not required.

\section{Consent for publication}

Not applicable.

\section{Availability of data and materials}

The dataset supporting the conclusions of this article is included within the article and its supplemental tables. Methylation and mRNA expression of ovarian cancer tissues were mined from The Cancer Genome Atlas (TCGA)(https://cancergenome.nih.gov/) and Gene expression omnibus (GEO) (https://www.ncbi.nlm.nih.gov/geo/)

\section{Competing interests}

The authors declare that the research was conducted in the absence of any commercial or financial relationships that could be construed as a potential conflict of interest. 


\section{Funding}

This work was supported by the grants from National Natural Science Foundation of China (No. 81871623).

\section{Authors' contributions}

Zhu S and Liu SL conceived and designed the study. Zhu S and Zhang M contributed to the computational analyses and confirmed the results. Zhu S and Bao H wrote the main manuscript text. Zhao X gave advice on the software applications. All authors have read and approved the final version of this manuscript.

\section{Acknowledgements}

Not applicable.

\section{References}

1. National Cancer Institute. SEER stat fact sheets: ovarian cancer. SEER [https://seer.cancer.gov/statfacts/html/ovary.html Released April 15, 2020]

2. Prat J: Pathology of cancers of the female genital tract. Int J Gynaecol Obstet 2015, 131 Suppl 2:S132-145.

3. O'Donovan PJ, Livingston DM: BRCA1 and BRCA2: breast/ovarian cancer susceptibility gene products and participants in DNA double-strand break repair. CARCINOGENESIS 2010, 31(6):961967.

4. Piek JM, Verheijen RH, Kenemans $\mathrm{P}$, Massuger LF, Bulten $\mathrm{H}$, van Diest PJ: BRCA1/2-related ovarian cancers are of tubal origin: a hypothesis. GYNECOL ONCOL 2003, 90(2):491.

5. Kulbe H, Otto R, Darb-Esfahani S, Lammert H, Abobaker S, Welsch G, Chekerov R, Schafer R, Dragun $D$, Hummel $\mathrm{M}$ et al: Discovery and Validation of Novel Biomarkers for Detection of Epithelial Ovarian Cancer. CELLS-BASEL 2019, 8(7).

6. Jiang Y, Lyu T, Che X, Jia N, Li Q, Feng W: Overexpression of SMYD3 in Ovarian Cancer is Associated with Ovarian Cancer Proliferation and Apoptosis via Methylating H3K4 and H4K20. J CANCER 2019, 10(17):4072-4084.

7. Zheng J, Zhou Y, Li XJ, Hu JM: MiR-574-3p exerts as a tumor suppressor in ovarian cancer through inhibiting MMP3 expression. Eur Rev Med Pharmacol Sci 2019, 23(16):6839-6848.

8. Harrington BS, Annunziata CM: NF-kappaB Signaling in Ovarian Cancer. Cancers (Basel) 2019, 11(8).

9. Mohamed NE, Hay T, Reed KR, Smalley MJ, Clarke AR: APC2 is critical for ovarian WNT signalling control, fertility and tumour suppression. BMC CANCER 2019, 19(1):677.

10. Matulonis UA, Sood AK, Fallowfield L, Howitt BE, Sehouli J, Karlan BY: Ovarian cancer. NAT REV DIS PRIMERS 2016, 2:16061. 
11. Kulis M, Esteller M: DNA methylation and cancer. ADV GENET 2010, 70:27-56.

12. Sincic N, Herceg Z: DNA methylation and cancer: ghosts and angels above the genes. CURR OPIN ONCOL 2011, 23(1):69-76.

13. Zhang W, Klinkebiel D, Barger CJ, Pandey S, Guda C, Miller A, Akers SN, Odunsi K, Karpf AR: Global DNA Hypomethylation in Epithelial Ovarian Cancer: Passive Demethylation and Association with Genomic Instability. Cancers (Basel) 2020, 12(3).

14. Mase S, Shinjo K, Totani H, Katsushima K, Arakawa A, Takahashi S, Lai HC, Lin RI, Chan MWY, Sugiura-Ogasawara $\mathrm{M}$ et al: ZNF671 DNA methylation as a molecular predictor for the early recurrence of serous ovarian cancer. CANCER SC/2019, 110(3):1105-1116.

15. Groot KR, Sevilla LM, Nishi K, DiColandrea T, Watt FM: Kazrin, a novel periplakin-interacting protein associated with desmosomes and the keratinocyte plasma membrane. J CELL BIOL 2004, 166(5):653-659.

16. Gallicano GI, Foshay K, Pengetnze Y, Zhou X: Dynamics and unexpected localization of the plakin binding protein, kazrin, in mouse eggs and early embryos. Dev Dyn 2005, 234(1):201-214.

17. Sevilla LM, Nachat R, Groot KR, Watt FM: Kazrin regulates keratinocyte cytoskeletal networks, intercellular junctions and differentiation. J CELL SCI 2008, 121(Pt 21):3561-3569.

18. Wang Q, Liu M, Li X, Chen L, Tang H: Kazrin F is involved in apoptosis and interacts with BAX and ARC. Acta Biochim Biophys Sin (Shanghai) 2009, 41(9):763-772.

19. Chhatriwala MK, Cipolat S, Sevilla LM, Nachat R, Watt FM: Exons 5-15 of kazrin are dispensable for murine epidermal morphogenesis and homeostasis. J INVEST DERMATOL 2012, 132(8):1977-1987.

20. Sevilla LM, Rana AA, Watt FM, Smith JC: KazrinA is required for axial elongation and epidermal integrity in Xenopus tropicalis. Dev Dyn 2008, 237(6):1718-1725.

21. Cho K, Vaught TG, Ji H, Gu D, Papasakelariou-Yared C, Horstmann N, Jennings JM, Lee M, Sevilla LM, Kloc $\mathrm{M}$ et al: Xenopus Kazrin interacts with ARVCF-catenin, spectrin and p190B RhoGAP, and modulates RhoA activity and epithelial integrity. J CELL SCI2010, 123(Pt 23):4128-4144.

22. Watanabe Y, Maekawa M: Methylation of DNA in cancer. ADV CLIN CHEM 2010, 52:145-167.

23. Hentze JL, Hogdall CK, Hogdall EV: Methylation and ovarian cancer: Can DNA methylation be of diagnostic use? Mol Clin Oncol 2019, 10(3):323-330.

24. Zhang Y, Parmigiani G, Johnson WE: ComBat-seq: batch effect adjustment for RNA-seq count data. NAR Genom Bioinform 2020, 2(3):Iqaa078.

25. Wu P, Heins ZJ, Muller JT, Katsnelson L, de Bruijn I, Abeshouse AA, Schultz N, Fenyo D, Gao J: Integration and Analysis of CPTAC Proteomics Data in the Context of Cancer Genomics in the cBioPortal. MOL CELL PROTEOMICS 2019, 18(9):1893-1898.

26. Yang $\mathrm{H}$, Wang $\mathrm{K}$ : Genomic variant annotation and prioritization with ANNOVAR and WANNOVAR. NAT PROTOC 2015, 10(10):1556-1566.

27. Nachat R, Cipolat S, Sevilla LM, Chhatriwala M, Groot KR, Watt FM: KazrinE is a desmosomeassociated liprin that colocalises with acetylated microtubules. J CELL SCI 2009, 122(Pt 22):4035- 
4041.

28. Liu C, Wang J, Hu Y, Xie H, Liu M, Tang H: Upregulation of kazrin F by miR-186 suppresses apoptosis but promotes epithelial-mesenchymal transition to contribute to malignancy in human cervical cancer cells. Chin J Cancer Res 2017, 29(1):45-56.

29. GTEx Transcript Browse.https://www.gtexportal.org/home/transcriptPage

30. Karpf AR, Matsui S: Genetic disruption of cytosine DNA methyltransferase enzymes induces chromosomal instability in human cancer cells. CANCER RES 2005, 65(19):8635-8639.

31. Houshdaran S, Nezhat CR, Vo KC, Zelenko Z, Irwin JC, Giudice LC: Aberrant Endometrial DNA Methylome and Associated Gene Expression in Women with Endometriosis. BIOL REPROD 2016, 95(5):93.

\section{Tables}

Table 1 Features of the enrolled Gene Expression Omnibus datasets

\begin{tabular}{|c|c|c|c|c|c|c|c|c|c|c|}
\hline \multirow{2}{*}{ Accession } & \multirow{2}{*}{ GPL } & \multirow{2}{*}{ Year } & \multicolumn{3}{|c|}{ Tumor } & \multicolumn{3}{|c|}{ Normal } & \multirow{2}{*}{ p-value } & \multirow{2}{*}{ Source } \\
\hline & & & $\mathrm{N}$ & $\mathrm{M}$ & SD & $\mathrm{N}$ & $\mathrm{M}$ & SD & & \\
\hline GSE105437 & GPL570 & 2017 & 10 & 1121.506 & 246.6307 & 5 & 734.7216 & 132.1731 & 0.001701071 & tissue \\
\hline GSE27651 & GPL570 & 2011 & 43 & 475.5898 & 279.5294 & 6 & 145.5886 & 45.25907 & $6.603179 \mathrm{e}-09$ & tissue \\
\hline GSE36668 & GPL570 & 2012 & 4 & 323.7239 & 23.08583 & 4 & 183.7234 & 52.44505 & 0.007529066 & tissue \\
\hline GSE66957 & GPL1 5048 & 2015 & 57 & 7.500341 & 0.5333843 & 12 & 6.60236 & 0.4617553 & $1.310033 \mathrm{e}-05$ & tissue \\
\hline GSE69428 & GPL570 & 2015 & 10 & 7.175477 & 0.9314753 & 10 & 6.292184 & 0.4273923 & 0.01771169 & tissue \\
\hline GSE29450 & GPL571 & 2011 & 10 & 7.175477 & 0.7607612 & 10 & 7.612039 & 0.4370185 & 0.05491613 & tissue \\
\hline GSE37582 & GPL6947 & 2012 & 74 & 5.669769 & 0.09359612 & 47 & 5.785942 & 0.1336951 & $1.673298 \mathrm{e}-06$ & blood \\
\hline
\end{tabular}

N Number of sample, M Mean, SD Standard deviation

\section{Figures}




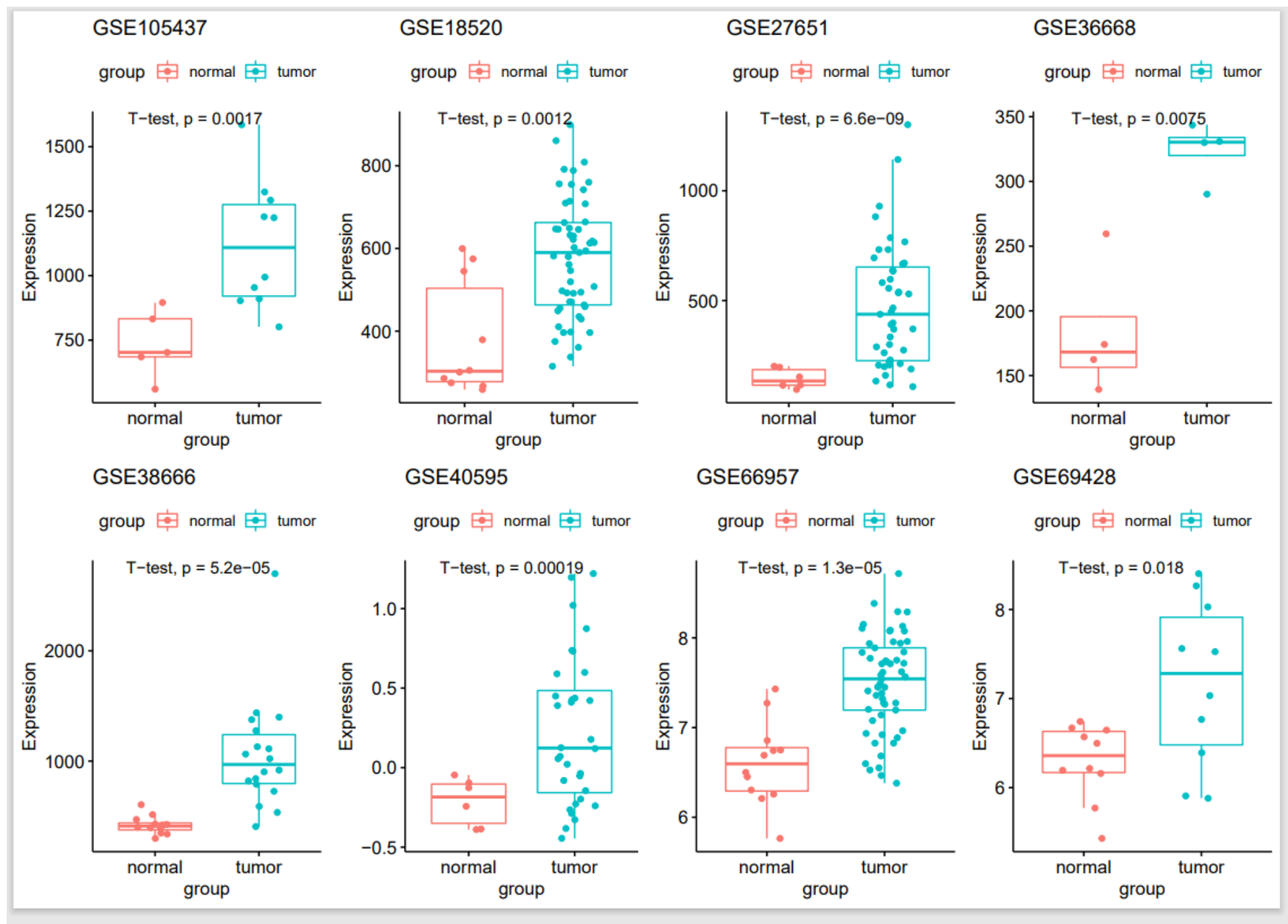

Figure 1

Expression of KAZN in ovarian cancer tissues and normal tissues based on Gene Expression Omnibus datasets. The expressions of KAZN are up-regulated in GEO datasets GSE105437, GSE18520, GSE27651, GSE36668, GSE38666, GSE40595, GSE66957, GSE69428. 


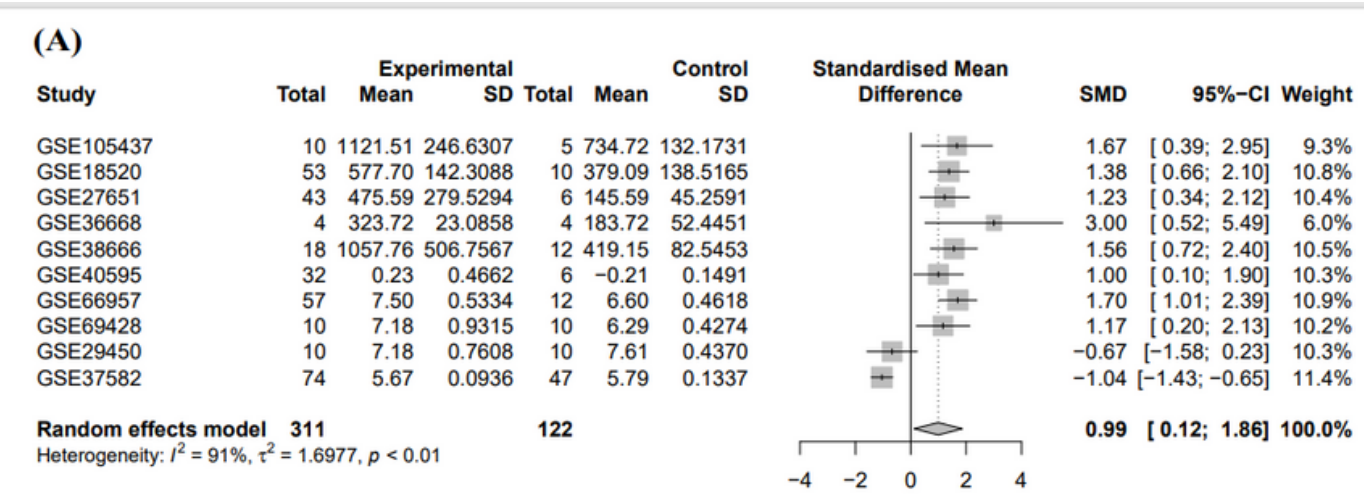

(B)

Study

Omitting GSE105437

Omitting GSE18520

Omitting GSE2765

Omitting GSE36668

Omitting GSE38666

Omitting GSE40595

Omitting GSE66957

Omitting GSE69428

Omitting GSE29450

Omitting GSE37582

Random effects model
Standardised Mean Difference

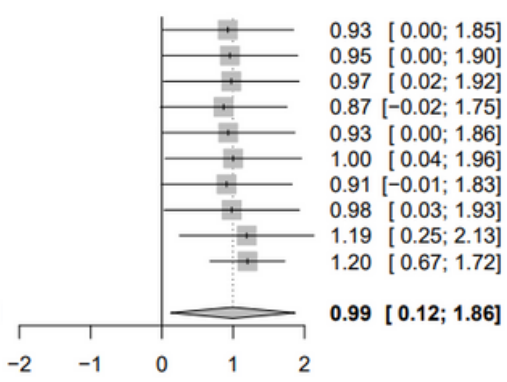

(C)

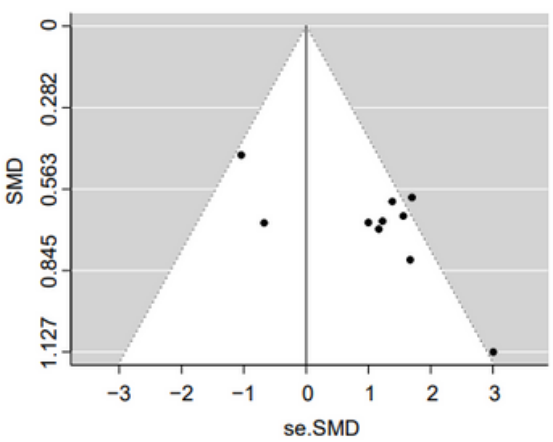

Figure 2

Meta-analysis of Gene Expression Omnibus (GEO) data. (A) Forest plot of GEO chips. The standard mean deviation is $0.99(95 \% \mathrm{Cl}: 0.12,1.86)$ with great heterogeneity $(\mathrm{I} 2=91 \%, \mathrm{p}<0.01)$ showed that KAZN expression is markedly up-regulated in the ovarian cancer tissues. (B) Sensitivity analysis of GEO chips. (C) A funnel plot was applied to evaluate the publication bias of GEO chips (Begg's test, $p=0.363$ ) 


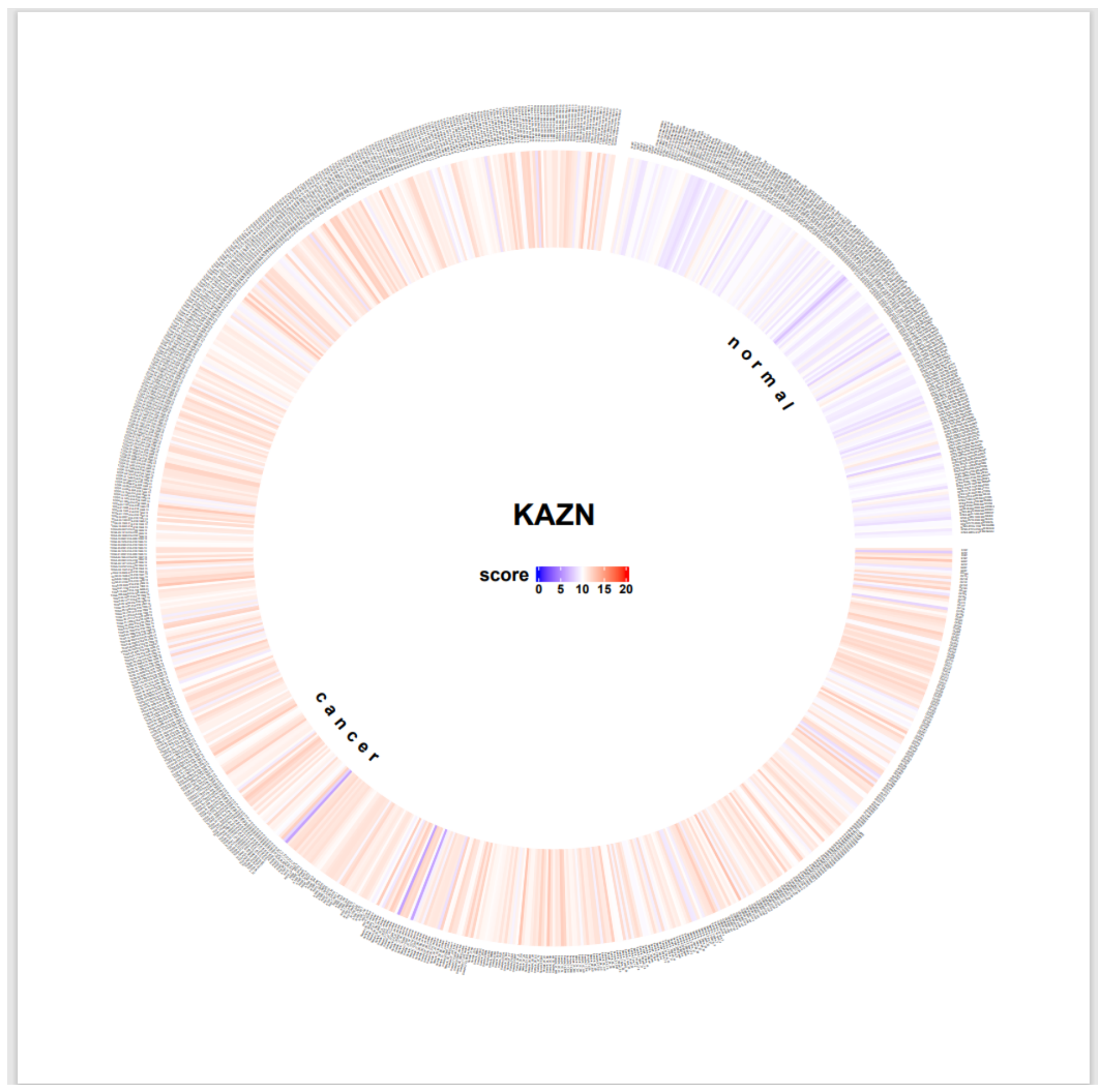

Figure 3

Circular heatmaps of KAZN expression based on TCGA, GTEx and GEO. 


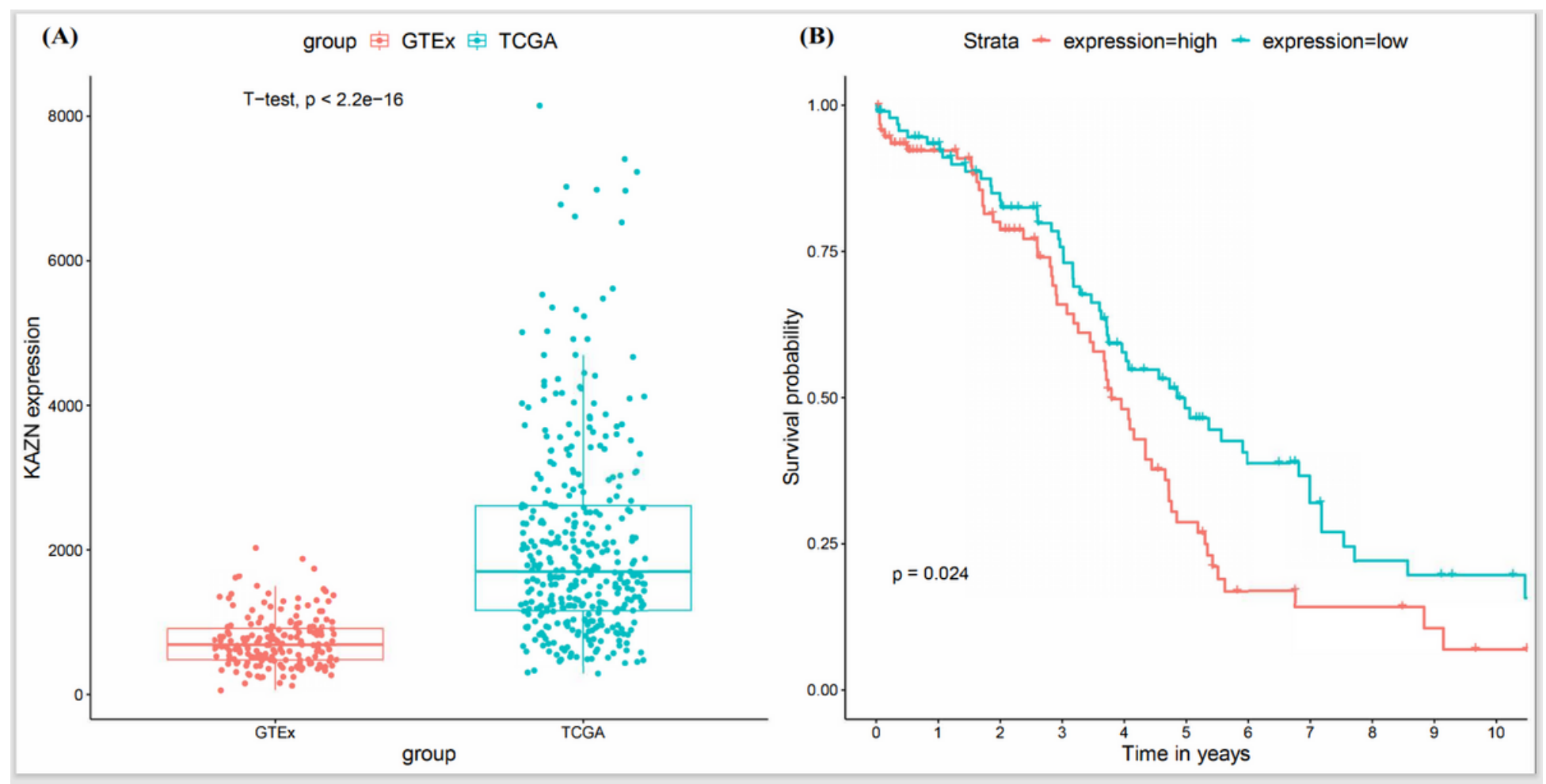

\section{Figure 4}

KAZN expression in ovarian cancer and survival analysis based on TCGA database. (A) The box plot illustrates median expression levels of the KAZN in normal ovarian tissue (Left, from GTEx) and ovarian tumors (Right, from TCGA). (B) The Kaplan-Meier survival curve illustrate overall survival for patients who had tumor with up-regulated or down-regulated KAZN. 


\section{group}

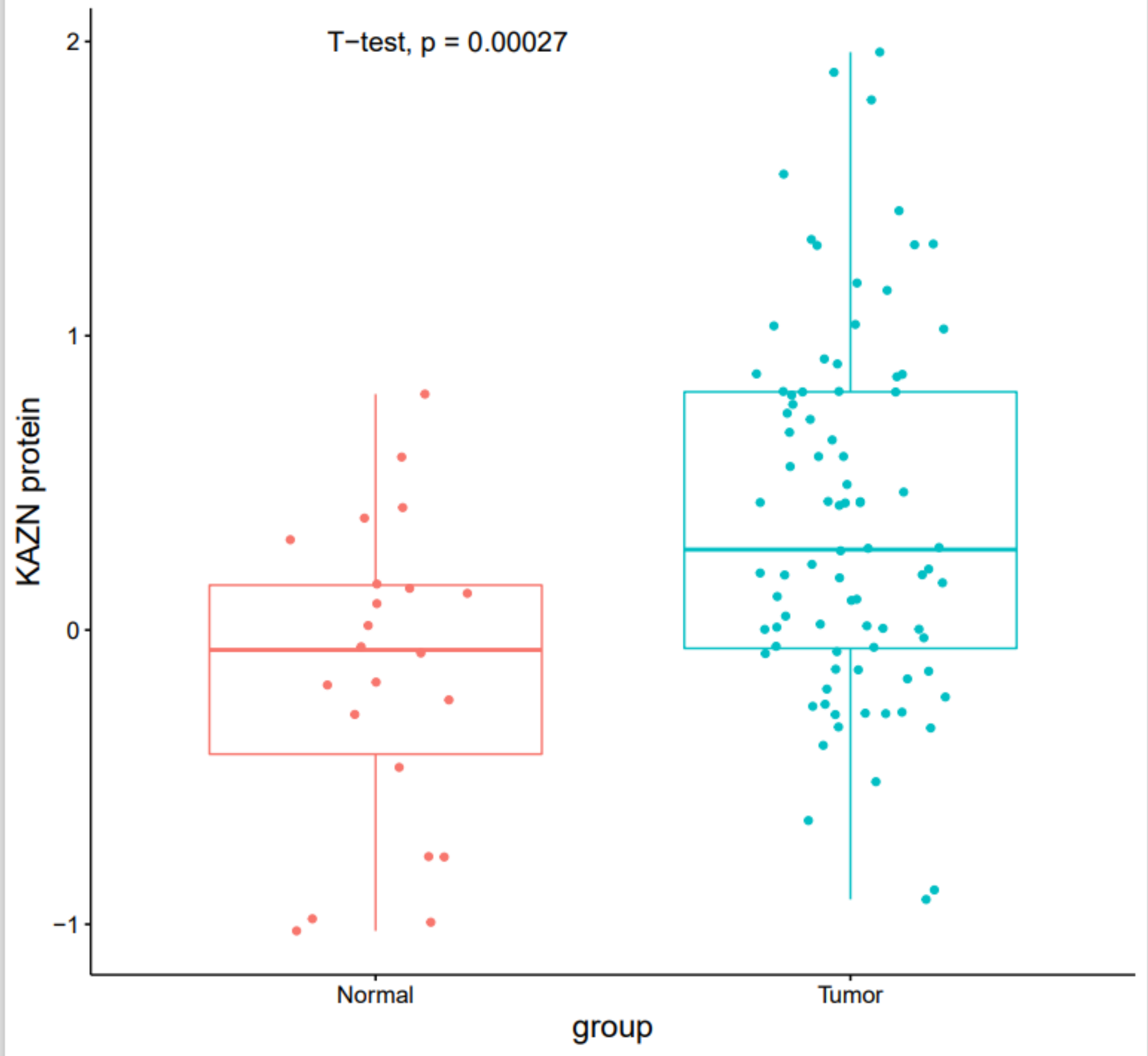

Figure 5

KAZN protein level in ovarian cancer. The box plot illustrates median of the KAZN protein in normal ovarian tissue (Left) and ovarian tumors (Right) based on CPTAC datasets. 


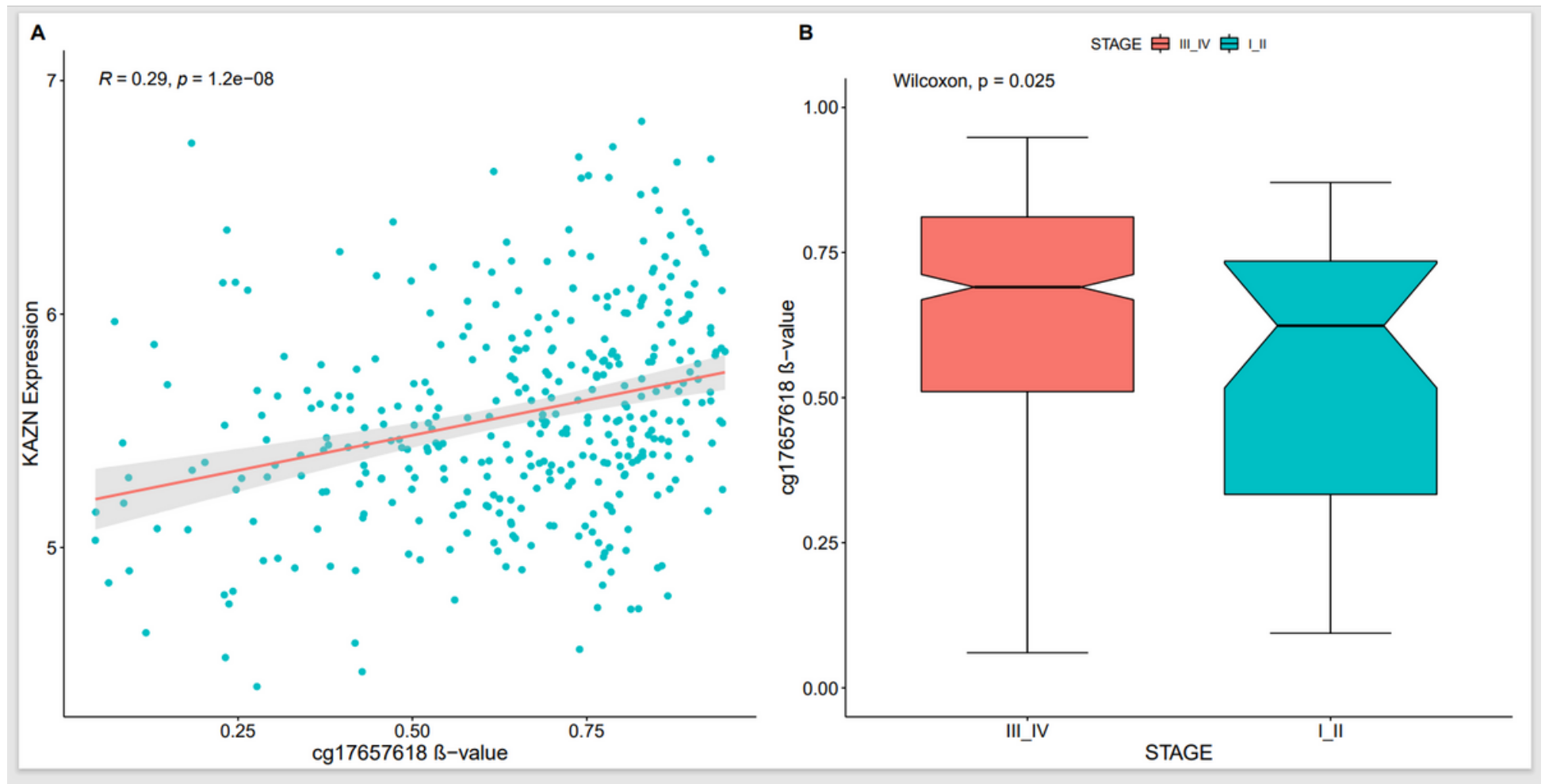

Figure 6

Correlation between KAZN methylation and mRNA expression and survival analysis. (A) This plot illustrates that KAZN mRNA is positively correlated to cg17957618 methylation level $(R=0.27, p=1.2 e-$ 08). (B) Boxplot for the cg17657618 methylation level between "stage I and II" group and "stage III and IV" group 


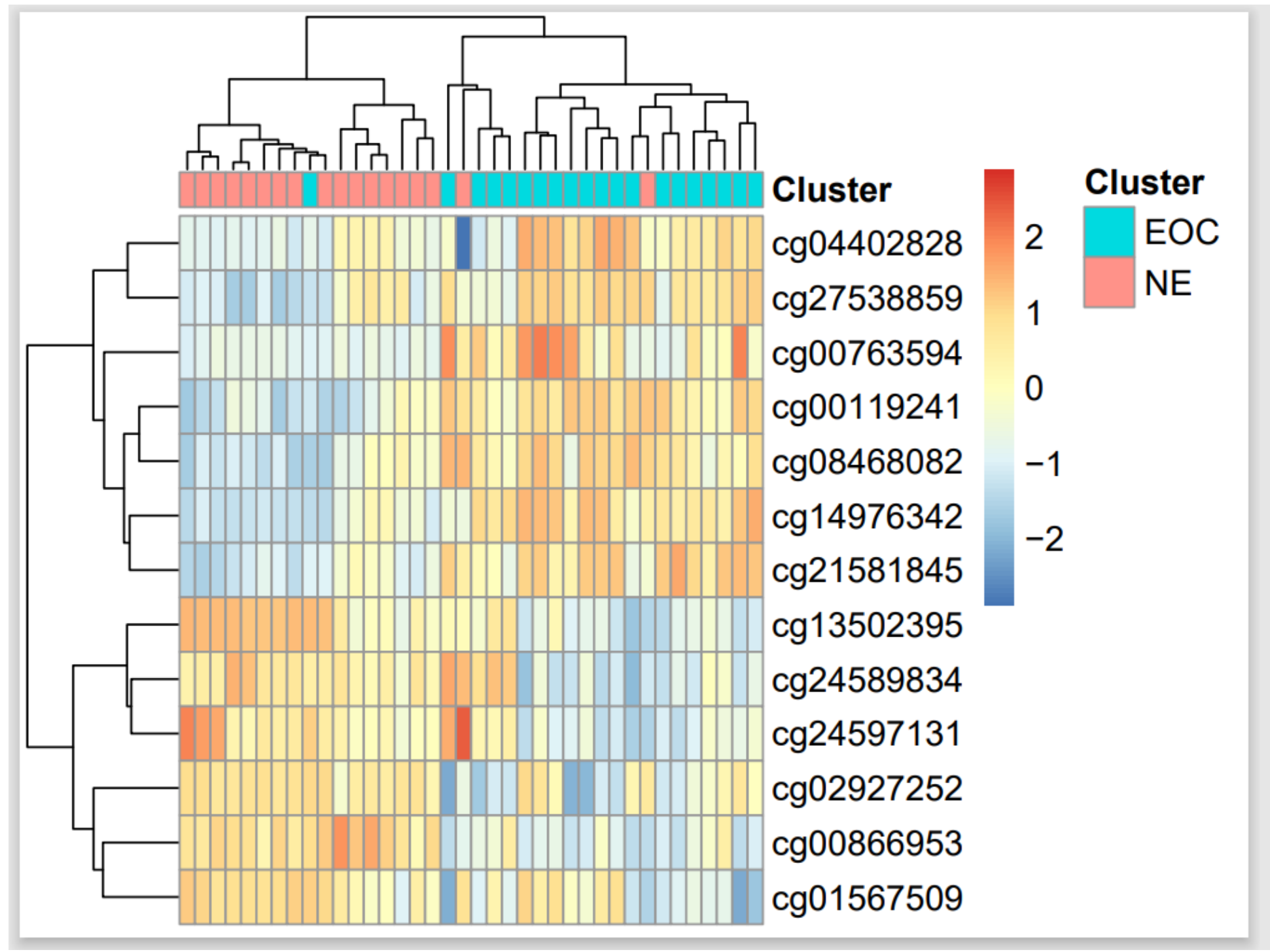

Figure 7

Pheatmap of 13 KAZN methylation CpG sites. EOC (epithelial ovarian cancer) group and NE (not epithelial ovarian cancer, includes epithelial layer of normal ovary and fimbriae fallopian tubes) group clustered together respectively. In the two groups have different methylation pattern.

\section{Supplementary Files}

This is a list of supplementary files associated with this preprint. Click to download.

- FigureS1.pdf

- Figures2.pdf

- SupplementaryMaterial.docx

- TableS1.pdf 